
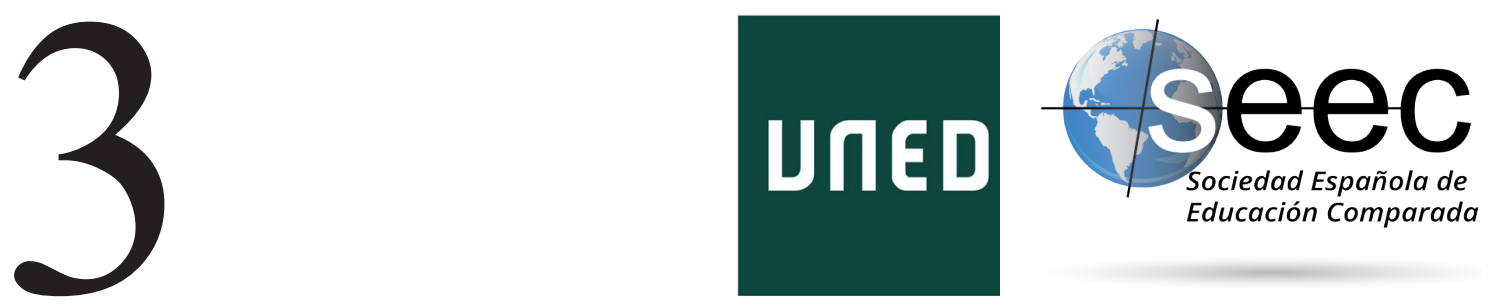

\title{
Educar a la ciudadanía global en una perspectiva intercultural
}

Educate global citizenry in an intercultural perspective

\section{Massimiliano Fiorucci*}

DOI: $10.5944 /$ reec.30.2017.20629

\author{
Recibido: 10 de diciembre de 2017 \\ Aceptado: 30 de diciembre de 2017
}

\footnotetext{
* Massimiliano Fionucci: Profesor Ordinario en el Departamento de Ciencias de la Formación de la Univesità degli Studi Roma Tre. Imparte Pedagogía Social e Intercultural. Coordina el Grado de Ciencias de la Educación, dirige el Master de Educación Intercultural. Es, también, Coordinador científico del Centro de Investigación CREIFOS (<www.creifos.org >). Sus líneas de investigación se centran en la pedagogía intercultural, con particular atención a la mediación intercultural y a la literatura de migrantes. También investiga: la educación y formación de personas adultas; la formación intercultural de docentes y educadores; la formación en las organizaciones y la pedagogía social. Datos de contacto: E-mail: massimiliano. fiorucci@uniroma3.it
} 


\title{
Resumen
}

Diferentes situaciones sociohistóricas han producido y seguirán produciendo desplazamientos de personas de unos territorios a otros. El sistema educativo está llamado a implicarse y a trazar posibles vías de actuación en una sociedad cada vez más plural y compleja, porque estos cambios, que ponen en tela de juicio los sistemas educativos y formativos establecidos en Europa, hacen que la sociedad dependa mucho más que antes de personas desconocidas que jamás se han visto entre sí y que deben afrontar cuestiones de carácter mundial (medioambientales, económicas, políticas y religiosas), que no podrán solucionarse por separado. En este ensayo se trata de aportar elementos contextualizadores indispensables en cualquier intento de abordar el cambio educativo. Las instituciones deben proporcionar nuevos conocimientos a los estudiantes sobre grupos minoritarios, sobre sus luchas, conquistas o su contribución a la cultura nacional, pero también de conocimientos referentes a otros países y sus poblaciones. Este conocimiento por sí solo no garantiza un comportamiento correcto, pero es requisito previo para superar estereotipos y prejuicios. Para comprender mejor la cuestión se proponen ejemplos referidos a Italia: su secular composición multicultural, la reducción de las humanidades y artes en favor de habilidades técnicas y conocimientos prácticos y científicos, y se analizan propuestas concretas de relevantes autores para una acción educativa intercultural. Se contrapone la educación orientada a la ganancia versus la educación hacia una ciudadanía más inclusiva, hacia la democracia teniendo en cuenta, además, que no es algo que atañe solo a la escuela, sino a toda la sociedad en sus distintos niveles: la integración social tiene que ver también con las políticas de integración que un país está dispuesto a poner en marcha. Se concluye que la educación intercultural no es una nueva disciplina a añadir, sino una perspectiva diferente con la que ver los conocimientos que se enseñan actualmente.

Palabras clave: Interculturalidad; Ciudadanía Global; Italia

\begin{abstract}
Various socio-historic situations have produced, and continue displacements of people from one territory to another. The educational system is compelled to involve and find possible ways of action in a society which is more and more plural and complex because these changes, that question educational and formative systems established in Europe, make society depend much more than before on unknown persons who have never met each other and who must face world issues (environmental, economic, political and religious), that cannot been solved separately. This essay tries to provide contextualising elements essential in any attempt to tackle changes in Education. Institutions must not only provide new knowledge to their students about minority groups, their struggles, their success or their contribution to national culture, but also knowledge regarding other countries and their peoples. This knowledge alone, does not guarantee correct behaviour, but it is an essential requirement to overcome stereotypes and prejudices. In order to better understand the question examples referring to Italy are put forward. It secular multicultural composition; the reduction of studies of humanities and arts in favour of technical skills and scientific and practical knowledge; and specific proposals from relevant authors are analysed in order to obtain an intercultural educative action. Education focused on profit versus education focused on inclusive citizenship for democracy is challenged; taking into account it is not something which only concerns schools but involves all society at several levels: social integration has much to do with the politics of integration that a country is disposed to set up. It is concluded that intercultural education is not a new subject to add in schools, but a different perspective with which to study current knowledge.
\end{abstract}

Key Words: Interculturality; Global Citizenry; Italy 


\section{Formar identidades abiertas y fomentar el pensamiento crítico}

La etapa histórica actual está experimentando grandes transformaciones de carácter socioeconómico y político, determinas por diferentes factores. Entre estos, los grandes procesos de movilidad humana que implican un número siempre mayor de contactos y relaciones entre personas de origen social y cultural diferentes, desempeñan un papel muy importante. Por lo tanto, es indispensable proyectar, elaborar y realizar caminos educativos y formativos que estén a la altura de los problemas actuales para entender los procesos en marcha sin tener que aguantarlos, sin que nos afecten y evitando que puedan causar el así llamado «choque de civilizaciones» (Huntington, 1996). Las guerras, las enormes disparidades en la distribución de los recursos, el desequilibrio entre los países ricos y pobres, las nuevas formas de colonialismo en marcha producen y producirán, independientemente de las opiniones, siempre más desplazamientos de personas. El sistema educativo y formativo está directamente implicado y la pedagogía tiene la tarea inaplazable de trazar las características fundamentales en la actual época del pluralismo y de la complejidad (Portera, 2006). En pocas palabras, se trata de construir condiciones para la positiva convivencia entre personas que se refieren a sistemas culturales y de valores hasta muy diferentes en algunos casos, que adquieren una perspectiva muy diferente respecto a la que Samuel P. Huntington imaginaba. «El miedo a los bárbaros - advierte Todorov- es lo que amenaza con convertirnos en bárbaros» (Todorov, 2008, p. 18). Construir las condiciones para una convivencia positiva entre las personas es, antes que nada, una cuestión educativa: la «cultura de convivencia» implica tanto a las mayorías como a las minorías. De hecho, los procesos de integración son tales solo si son duales y bilaterales.

Se están verificando cambios históricos que ponen en tela de juicio los sistemas educativos y formativos: dependemos mucho más que antes de las personas que no conocemos y que jamás hemos visto y viceversa. Las cuestiones que debemos afrontar (medioambientales, económicas, políticas y religiosas) son de carácter mundial y no podrán solucionarse si las «personas que se encuentran distantes no se unen para cooperar como jamás lo han hecho» (Nussbaum, 2010, p. 114). pensemos, solo para hacer un ejemplo, al calentamiento global, a la protección del medio ambiente, a las cuestiones energéticas, a la definición de dignas condiciones laborales, etc. Dichas cuestiones podrán solucionarse positivamente solo en una perspectiva supranacional, hasta empezando por la reconsideración de los modelos educativos y de educación: nadie puede considerarse ajeno a esta interdependencia mundial. Nuestras decisiones de consumo, así como aquellas medioambientales producen consecuencias decisivas en las condiciones de vida y de trabajo de otras personas que viven en países lejanos y, entonces, una de las tareas más urgentes y prioritarias de la escuela y de la Universidad es la de concienciarnos de ello, ayudándonos a considerarnos como miembros de la más amplia comunidad humana, superando una perspectiva nacionalista y autorreferencial improductiva y dañina.

Son sobre todo las instituciones las que tienen la tarea de proporcionar nuevos conocimientos a los estudiantes: se trata de conocimientos referentes a grupos minoritarios y a los subgrupos (religiosos, de género, minorías lingüístico-culturales, etc.) a sus luchas, a sus conquistas, a su contribución a la cultura nacional, pero también de conocimientos referentes a otros países y a sus poblaciones. El conocimiento por sí solo no garantiza 
un comportamiento correcto, sino que representa el requisito previo para superar los estereotipos y los prejuicios más banales y más superficiales. «Para empezar a combatir esos estereotipos es necesario procurar que, desde una edad muy temprana, los niños y las niñas aprendan a relacionarse de otra manera con el mundo, contando con datos correctos y valiéndose de una curiosidad respetuosa» (Nussbaum, 2010, p. 115).

La tarea de enseñar una ciudadanía inteligente del mundo parece tan grande que uno está tentado de rendirse y declarar que sería mejor encargarnos solo de nuestro país (Nussbaum, 2010), pero hasta solo estudiar y entender a su propio país implica el conocimiento de los grupos y de las culturas que la componen. Algunos ejemplos que se refieren a Italia pueden ayudar a una mejor comprensión de la cuestión. Antes que nada, no debe olvidarse que la cultura de un país es también el resultado de una construcción, puesto que se basa en la memoria colectiva del grupo que la posee y la memoria es, en sí misma, construcción, es decir la selección de los acontecimientos del pasado y su jerarquización según un orden que le conceden sus mismos miembros. Cada comunidad humana -a través de la acción de determinados grupos de poder- selecciona algunos sucesos y deja otros a un lado: tanto la memoria como el olvido son elementos que constituyen la identidad. De hecho, hay grupos, fuerzas políticas, periodistas, historiadores, organismos individuales y asociaciones que, por diferentes razones, no se reconocen dentro de la historia oficial que, por su naturaleza, es un relato que debe, necesariamente, hacer una selección entre diferentes acontecimientos históricos y ordenarlos de manera jerárquica.

Este proceso es aún más evidente cuando un país intenta volver a construir históricamente las relaciones con las alteridades exteriores (otros países) e interiores (minorías lingüísticas, culturales y religiosas). Siempre refiriéndonos a Italia, para poner ejemplos concretos, sería muy necesario volver a considerar con oportunas herramientas críticas y de análisis, por un lado la historia y los horrores del colonialismo italiano en África ${ }^{1}$ y por otro los comportamientos hacia particulares grupos o minorías, con particular referencia a las «leyes raciales fascistas» de 1938 dirigidas a los judíos y, recientemente, a la exclusión de las minorías romaní y sinti de la Ley del 15 de diciembre de 1999, n. 482 «Normas relativas a la protección de las minorías lingüísticas históricas». Por supuesto, se trata de sucesos y medidas muy diferentes entre sí y con diferente valor jerárquico y que, sin embargo, contribuyen a comprender cuál ha sido y cuál es el lugar que una sociedad y un país atribuyen a las así llamada alteridades. Por lo tanto, se vuelve esencial la capacidad de descentralización, en pocas palabras:

«lo que debe valorarse es el momento en que el individuo toma consciencia de la identidad de su grupo y es capaz de observarlo como desde el lugar de otro, ya que así tiene la posibilidad de escrutar con mirada crítica su pasado para reconocer en él los antiguos rasgos tanto de humanidad como de barbarie. No podemos conocer las propias tradiciones y la propia cultura si no sabemos tomar cierta distancia respecto de ellas, lo que en absoluto debe confundirse con la autodenigración sistemática y la fustigación pública, pero tampoco con la tranquila seguridad de haber tenido siempre razón. Se trata más bien de sustituir los gritos de orgullo y las lágrimas de arrepentimiento por el cuestionamiento sobre las causas y el sentido de los acontecimientos pasados» (Todorov, 2008: p. 87).

1 Durante la larga e importante, pero descuidada, experiencia colonial italiana se «experimentaron» verdaderas formas de violencia sistemática (utilización de gases, campos de concentración, leyes raciales) que contradicen la representación tranquilizadora y autoabsolutoria de los «italianos buena gente» (Del Boca, 2005). 
También con referencia a su propia autorepresentación como país multicultural, el caso italiano merece unas cuantas reflexiones. En los discursos públicos y en la comunicación masiva, parece como si antes del fenómeno migratorio Italia hubiese sido un país lingüísticamente y culturalmente homogéneo. En realidad, no es así. A nivel internacional Italia se presenta con una historia migratoria muy particular. Las grandes migraciones del siglo XX incluyeron a Italia en una triple perspectiva: en principio como tierra de emigración (aproximadamente 26 millones de emigrantes en cien años: 1873-1973; más de sesenta millones de oriundos italianos en el mundos y en la actualidad 4.636.647 ciudadanos italianos residentes en el extranjeros inscritos en el AIRE (Registro de los Italianos Residentes en el Extranjero en el $1^{\circ}$ de enero de 2015) (Fundación Migrantes 2015), después como país de inmigración (en el año 1973 se registró, por primera vez, una migración neta positiva) y a la vez como lugar de intensas y constantes migraciones internas. Los ciudadanos extranjeros regularmente presentes en Italia son, según la estimación del Dossier Estadístico italiano sobre Inmigración 2015, alrededor de 5 millones (5.421.000). Hay personas que vienen de diferentes partes del mundo, con diferentes porcentajes, y, sin embargo, los primeros cinco países de proveniencias son Rumania (1.131.839), Albania (490.883), Marruecos (449.058), China (265.820) y Ucrania (226.060) cuyos ciudadanos representan aproximadamente la mitad de los inmigrantes presentes en Italia (Idos-UNAR ${ }^{2}, 2015$ ).

Este flujo migratorio ha tenido también fuertes repercusiones en el sistema escolar, en menos de veinte años el número de estudiantes extranjeros ha aumentado en más de diez veces, pasando de 59.389 unidades (año escolar 1996-97) a 805.800 (año escolar 2013-14) con un porcentaje de niños y jóvenes de ciudadanía italiana que alcanzó el 9,2 \% (MIUR-ISMU 3 , 2015). Los segmentos escolares con la incidencia más alta, en términos porcentuales, son aquellos de la escuela primaria (10,3\%) y de la escuela de la infancia $(10,2 \%)$, seguidos por el primer ciclo de la escuela secundaria (9,6 \%) y por el segundo ciclo de la escuela secundaria $(7 \%)^{4}$. Las cosas cambian si se considera la presencia de niños y jóvenes en términos absolutos: escuela primaria (288.620), segundo ciclo de la escuela secundaria (185.877), escuela de la infancia (165.862) y primer ciclo de la escuela secundaria (165.441). Los niños y jóvenes extranjeros nacidos en Italia (las así llamadas segundas generaciones) que resultan matriculados en los colegios, constituyen la mayoría $(51,7 \%)$ de los alumnos con ciudadanía no italiana.

Además, cabe señalar que en Italia hay muchas minorías lingüísticas y culturales (Campani, 2008). Según las estimaciones del Ministerio del Interior italiano, alrededor del $5 \%$ de la población italiana tiene como lengua materna una lengua diferente del italiano. En su Constitución, la República italiana protege de forma explícita las minorías lingüísticas. El art.6 de la Constitución italiana («La República protegerá mediante normas adecuadas a las minorías lingüísticas») se aplicó antes de 1999 sobre todo en algunas regiones con estatuto especial (Valle de Aosta, Trentino-Alto Adigio, Friuli-Venecia Julia). En seguida, con la Ley n. 482/99 llamada «Normas en materia de tutela de las minorías lingüísticas históricas», se reconoció la existencia de otras minorías lingüísticas

2 Idos es el Dossier Estadístico de Inmigración italiano y UNAR es la Oficina Nacional Antidiscriminaciones Raciales de Italia.

3 El MIUR es el Ministerio de Educación y de Universidades e Investigación de Italia e ISMU se refiere a las Iniciativas y Estudios sobre la Multietnicidad.

4 En Italia la Enseñanza Primaria se dirige a niños de 6 a 10/11 años, el primer ciclo de la Escuela Secundaria a los de 11 a 13/14 años mientras el segundo ciclo de la Escuela Secundaria se destina a jóvenes de 14 a 18/19 años. 
(pero no de todas). Refiriéndose al art.6 constitucional, en el art.2 está escrito que «la Ley en efecto compromete a la República a la tutela de la lengua y cultura de las poblaciones albanesas, catalanas, germánicas, griegas, eslovenas, croatas y de las que hablan el francés, el franco-provenzal, el friulano, el ladino, el occitano y el sardo». Además, la Ley contiene normas específicas para la enseñanza de las lenguas minoritarias en las escuelas de las 12 comunidades lingüísticas reconocidas. Las instituciones escolares, según la Ley, deben asegurar la enseñanza de las lenguas de minoría y se les reconoce el derecho, a los que pertenecen a dichas minorías, a aprender su idioma materno. En particular, los art. 4 y 5 de la Ley n. 482/99 prevén intervenciones tanto a nivel de las instituciones escolares individuales como a nivel del Ministerio de Educación y de Universidades e Investigación.

Pero una de las minorías que fue excluida por la ley es aquella de los grupos romaníes y sintis. De hecho, las comunidades romaníes y sintis no fueron mencionadas ni en el Primer Informe sobre la condición de las minorías en Italia, redactado por la Oficina Central para los Problemas en las Zonas de Frontera y de las Minorías Étnicas del Ministerio del Interior (1994), ni en la Ley n. 482/99 ya mencionada. Sin embargo, cabe señalar que, en febrero de 2011, la Comisión Extraordinaria para la Protección y el Fomento de los Derechos Humanos del Senado de la República italiana (Legislatura XVI) hizo públicos los resultados de la investigación sobre la condición de los romaníes, sintis y del grupo gitano siciliano llamado caminanti, publicando el denominado Informe Final de la investigación sobre la condición de romaníes, sintis y caminanti en Italia. Se trata de un documento muy importante, puesto que, por primera vez, esta cuestión adquirió categoría nacional.

Por lo tanto, parece totalmente inadecuada la formación del ciudadano «italiano» que ha sido la función tradicional de sistema escolar: pero ¿Cómo es posible garantizar una formación a la altura de los retos del presente? ¿Cómo es posible educar a la ciudadanía mundial en una etapa de crisis de la educación durante la cual disminuyen de manera progresiva las inversiones destinadas al sistema de la educación?

Según Martha Nussbaum, para mantener viva la democracia es necesario invertir aquella orientación que ha llevado, en estos últimos años, a la reducción de las financiaciones destinadas a los estudios humanísticos y artísticos en favor de habilidades técnicas y conocimientos prácticos y científicos. Atraídos por la idea de la ganancia a corto plazo, los países están progresivamente disminuyendo los programas de estudio de tipo humanístico en las escuelas y en las Universidades, consideradas poco útiles, en favor de conocimientos técnicos y científicos más aptos al objetivo: mientras el mundo se vuelve siempre más complejo, las herramientas para entenderlo se vuelven más pobres y rudimentarias.

«Las naciones - afirma Nussbaum - cada vez se sienten más atraídas por la idea del beneficio; ellas y sus sistemas escolares están acantonando, sin criterio alguno, unos saberes que son indispensables para mantener viva la democracia. Si esta tendencia se alarga, bien pronto los países de todo el mundo producirán generaciones de máquinas dóciles en lugar de ciudadanos dignos de ese nombre, capaces de pensar por sí mismos y de criticar a otros. El futuro de las democracias de todo el mundo pende de un hilo» (Nussbaum, 2010: pp. 22-23). 
Según Nussbaum, no se trata de defender una presunta superioridad de la cultura clásica sobre aquella científica, sino de mantener el acceso hacia aquel conocimiento que alimenta la libertad de pensamiento y de palabra, la autonomía del juicio, la capacidad de pensar críticamente, la capacidad de superar localismos y afrontar los problemas mundiales como "ciudadanos del mundo» y la capacidad de representarse de manera comprensiva con la categoría de los demás. Entonces, la contraposición no es entre la cultura humanística y la cultura científica, sino entre la educación orientada a la ganancia y la educación hacia una ciudadanía más inclusiva (para la democracia), como también demuestran muy bien Nuccio Ordine (2013) y Abraham Flexner (2013) en sus consideraciones sobre la utilidad de los conocimientos inútiles.

De todas formas, hay conocimientos sin un fin en sí mismos que, precisamente debido a su naturaleza gratuita y desinteresada, alejados de todo tipo de limitación práctica y comercial, pueden desempeñar un papel fundamental en el cultivo del espíritu y en el crecimiento civil y cultural de la humanidad (Ordine, 2013). Lamentablemente,

«la lógica del beneficio mina por la base las instituciones (escuelas, universidades, centros de investigación, laboratorios, museos, bibliotecas, archivos) y las disciplinas (humanísticas y científicas) cuyo valor debería coincidir con el saber en sí, independientemente de la capacidad de producir ganancias inmediatas o beneficios prácticos. Es cierto que con mucha frecuencia los museos o los yacimientos arqueológicos pueden ser también fuentes de extraordinarios ingresos. Pero su existencia, contrariamente a lo que algunos querrían hacernos creer, no puede subordinarse al éxito económico: la vida de un museo o una excavación arqueológica, como la de un archivo o una biblioteca, es un tesoro que la colectividad debe preservar con celo a toda costa» (Ordine, 2013, p. 6).

Martha Nussbaum marca un programa extremadamente exigente de formación moral en el que son, de la misma manera, importantes tanto los contenidos como los métodos pedagógicos activos, refiriéndose a una tradición occidental que, recuperando la extraordinaria actualidad de la pedagogía socrática, va de Rousseau a Dewey, una formación que no consiste en la asimilación pasiva de sucesos y tradiciones culturales, sino en la problematización, en acostumbrar la mente a volverse activa, competente y responsablemente crítica hacia la complejidad del mundo. He aquí —según Nussbaum (2010) - lo que puede hacer la escuela para formar a ciudadanos de una democracia sana.

- Desarrollar la capacidad de los estudiantes de ver el mundo desde el punto de vista de otras personas, en particular de aquellos que la sociedad tiende a representar como inferiores, como «meros objetos».

- Enseñar a enfrentarse con las insuficiencias y fragilidades humanas, es decir enseñar que la debilidad no debe ser la causa de vergüenza y que necesitar a los demás no es falta de virilidad; enseñar a los niños a que no se avergüencen de las necesidades y de las dificultades, sino a que vean todo ello como ocasión de cooperación y reciprocidad.

- Desarrollar la capacidad de una auténtica sensibilidad hacia los demás, que estén cerca o lejos. 
- Contrarrestar la tendencia de retirarse de las minorías, que por alguna razón se menosprecian, considerándolas «inferiores» $\mathrm{y}$ «contaminantes».

- Enseñar cosas auténticas sobre diferentes grupos (sobre las minorías raciales, religiosas y sexuales; sobre las personas con discapacidad), para rechazar los estereotipos y la repugnancia que a menudo los acompañan.

- Animar la responsabilidad, tratando a todo niño como un ser fiable.

- Fomentar firmemente el pensamiento crítico, la capacidad y el coraje que se necesita para levantar una voz discrepante.

El pensamiento crítico, autónomo, no homologado es, en pocas palabras, por sí solo intercultural, porque rechaza el prejuicio y el conocimiento ya listo, somete las opiniones al examen del conocimiento científico fundado, poniendo al individuo en condiciones de actuar y pensar libremente y de no ser fácilmente influido. Por lo tanto, Martha Nussbaum insiste en una pedagogía socrática que pone el razonamiento en el centro: el método socrático

«es particularmente crucial para la buena ciudadanía en una sociedad que tiene que luchar a brazo partido con la presencia de personas que difieren según la etnia, la casta, la religión. La idea de que cada uno asuma la responsabilidad de su propio razonamiento e intercambie ideas con otros en un ambiente de respeto mutuo a la razón, es esencial para la resolución pacífica de diferencias, tanto dentro de un país como de un mundo cada vez más polarizado por el conflicto étnico y religioso». (Nussbaum, 2010, p. 71).

Por lo tanto, el socrático es un método crítico que John Dewey reinterpretó con maestría, con el objetivo de formar a ciudadanos activos, curiosos, críticos y mutuamente respetuosos, que en sus experiencias educativas han aprendido a analizar, examinar, solucionar los problemas. Se trata de una perspectiva pedagógica indispensable para enfrentarse a los problemas del presente, el así llamado «mundo de las interdependencias» respecto al que nadie puede sentirse ajeno. La misma inmigración representa uno de los efectos más visibles en nuestras sociedades de la interdependencia mundial, y su comprensión significa interesarse a los problemas que los originan. Ya no es más posible estudiar la historia de un país sin situarlo en un contexto mundial. Es suficiente detenerse a pensar en las causas de los procesos de migración o hasta en el origen de los más comunes productos de consumo (del café a los alimentos, del vestuario a las bebidas) para tener que interrogarnos sobre las condiciones de vida y de trabajo en otros países en el mundo 5 . Se trata de enseñar lo que Edgar Morin definió como identidad terrenal. De hecho, basta poco para darse cuenta de los procesos de globalización en marcha, empezando por nuestros hábitos diarios:

$5 \quad$ Un ejemplo importante en esta dirección está representado por el comercio justo. El comercio justo, o fair trade, es un partenariado económico que se basa en el diálogo, la transparencia y el respeto, cuyo objetivo es una mayor equidad entre el Norte y el Sur del mundo, a través del comercio internacional. El comercio justo contribuye a un desarrollo sostenible total a través de la oferta de mejores condiciones económicas y asegurando los derechos para los productores marginados por el mercado de los trabajadores, en particular en el Sur del mundo. Por lo tanto, es una forma de comercio internacional en el que se intenta hacer crecer a empresas económicamente sanas y garantizar a los productores y a los trabajadores de los países en vías de desarrollo, un trato económico y social justo; en este sentido, se contrapone a las prácticas de comercio basadas en la explotación a menudo llevada a cabo por las empresas multinacionales que actúan exclusivamente con vistas a la maximización del beneficio. 
«Así, el europeo, por ejemplo, se despierta cada mañana prendiendo su radio japonés y recibe los acontecimientos del mundo: erupciones volcánicas, terremotos, golpes de Estado, conferencias internacionales le llegan mientras toma su té de Ceilán, India o China a menos que sea un café moka de Etiopía o un café arábigo de América Latina; se pone su camiseta, sus calzoncillos y su camisa de algodón de Egipto o de India; viste saco y pantalón de lana de Australia, tratada en Manchester luego en RoubaixTourcoing, o bien una chamarra de piel traída de China y unos pantalones de mezclilla estilo USA. Su reloj es suizo o japonés. Sus lentes son de carey ecuatoriano. Puede encontrar en su despensa de invierno fresas y cerezas de Argentina o de Chile, ejotes frescos de Senegal, aguacates o piñas de África, melones de la Isla de Guadalupe. Tiene sus botellas de ron de Martinica, vodka ruso, tequila mexicano, whisky americano. Puede escuchar en su casa una sinfonía alemana dirigida por un director de orquesta coreano a menos que asista ante la pantalla de su televisión a La Bohemia con la negra Barbara Hendricks, en el papel de Mimí y el español, Plácido Domingo, en el papel de Rodolfo.

Mientras que el europeo está en este circuito planetario de comodidad, un gran número de africanos, asiáticos, sudamericanos están en un circuito planetario de miseria. Sufren en su vida cotidiana los contragolpes del mercado mundial que afectan los precios del cacao, del café, del azúcar, de las materias primas que producen sus países. Han sido expulsados de sus pueblos por procesos mundializados provenientes del Occidente, principalmente los progresos del monocultivo industrial; campesinos autosuficientes se han vuelto suburbanos en busca de un salario; sus necesidades se traducen en lo sucesivo en términos monetarios. Aspiran a la vida del bienestar a la cual los hacen soñar la publicidad y las películas occidentales. Utilizan vajillas de aluminio o de plástico, beben cerveza o Coca Cola. Se acuestan sobre restos de colchonetas de espuma de poliestireno y visten playeras con estampados a la americana. Bailan al son de músicas sincréticas donde los ritmos de su tradición entran en una orquestación venida de Norteamérica. Así, para lo mejor y lo peor, cada humano, rico o pobre, del Sur o del Norte, del Este o del Oeste, lleva en él, sin saberlo, el planeta entero. La mundialización es a la vez evidente, subconsciente, omnipresente» (Morin, 2001, p. 35).

Si la globalización es patente hasta en nuestra vida cotidiana, aún debe conocerse mejor estudiando las relaciones entre las economías y las mutuas historias, donde han desempeñado un papel importante el colonialismo y, en tiempos más recientes, las sociedades multinacionales. Historia, geografía, economía, literatura, religión no pueden más enseñarse en una perspectiva solo local o como mucho nacional, porque no ayudan a entender la complejidad del problema del presente y del futuro. Por lo tanto, una educación a la ciudadanía mundial requiere aprender al menos las nociones básicas de la historia mundial, con una atención particular a las relaciones de poder, a las relaciones de dominio, a las relaciones entre los géneros y al papel de las minorías.

Pero hoy en día, una formación a la ciudadanía mundial no se requiere solo en la escuela, sino también en la sociedad en sus distintos niveles. Muchos profesionales y operadores se formaron en su propio trabajo, sin una perspectiva intercultural. Pero los fenómenos migratorios ponen de manifiesto de inmediato los efectos de la globalización 
en la vida cotidiana de todos nosotros. A lo largo de unos 20 años, la población inmigrante en Italia ha aumentado casi 20 veces, hasta llegar - como se dijo- a casi cinco millones de personas. Se trata, como es obvio, de cambios repentinos e históricos que obligan a que Administraciones, servicios, estructuras públicas y privadas cambien y se reajusten en una perspectiva intercultural. Sin embargo, para que los servicios sean eficaces, deben necesariamente diferenciar sus propios instrumentos, reconociendo que los inmigrantes no son un grupo social homogéneo, sino un conjunto bastante heterogéneo de personas que piden una desestandarización de las respuestas. Muy a menudo puede verse, por parte de los servicios, la dificultad de liberarse de una visión y posición «etnocéntrica» que, esencialmente, lleva a la petición de una integración concebida como asimilación; a veces, dicha condición parece ser determinada por la «rigidez estructural» de algunos servicios, equilibrada por la flexibilidad personal y profesional de los operadores que ponen a disposición grandes competencias y capacidades adquiridas principalmente a través de un compromiso personal e individual. Pero la innovación y las competencias no pueden encargarse a los operadores individuales, deben volverse patrimonio de las estructuras y de todos los que en ellas trabajan. La realidad social, siempre más compleja y fragmentada, la multiplicación de las necesidades y de las demandas han dejado claro que un servicio no es un producto estándar generado según las capacidades técnicas del operador, y tampoco una respuesta programada según los objetivos, sino un proceso que nace de la relación con el usuario. Las necesidades tienen una naturaleza compleja y requieren intervenciones flexibles, capaces de involucrar recursos, profesionalidad y competencias diferentes.

Además, los procesos de estructuralización de la inmigración en Italia, así como en España y en Europa, han complicado aún más este marco; de hecho, los usuarios inmigrantes se han vuelto cada vez más visibles a nivel familiar también:

- sigue aumentando el número de niños en las guarderías, en las escuelas, en los servicios pediátricos y en lugares para pasar el tiempo libre;

- aumenta el número de mujeres en las salas de partos, en los consultorios y en los servicios para la maternidad;

- en el territorio aparecen asociaciones de inmigrantes que se vuelven interlocutores de las instituciones;

- aumenta considerablemente el número de inmigrantes ancianos.

- Además, las exigencias de los inmigrantes son muy diferentes entre sí y debidas a:

- las diferencias individuales;

- las condiciones socioeconómicas;

- las características sociales y demográficas;

- las diferentes zonas de origen;

- los diferentes proyectos de migración (a corto, a medio y a largo plazo);

- las condiciones jurídicas y administrativas;

- los tiempos de llegada. 
En cambio, en los servicios sociales y sanitarios, en las escuelas y en el ámbito socioeducativo los operadores sientes la creciente exigencia de adquirir competencias interculturales. Son también diferentes las áreas en las que la acción intercultural se realiza, desde la esfera social a la escuela, de las organizaciones al territorio. En lo social, la competencia intercultural permite enfrentarse a diferentes situaciones y problemas, como las dificultades y los conflictos de convivencia, la seguridad, la rehabilitación urbana (casas, barrios) y el trabajo. En la escuela se desempeña el papel de diseñar tareas de acogida, inserción, enseñanza de la Segunda lengua o de la lengua étnica, se fomenta la comprensión de las diferencias culturales y la prevención de los prejuicios. Por último, en las organizaciones, se enfrentan las problemáticas relacionadas con los temas críticos de la comunicación intercultural dentro y fuera de la estructura (Santerini y Reggio, 2007).

Por lo tanto, se trata de poner en marcha estrategias y programas de formación cuyo objetivo es de volver a estructurar los servicios en una perspectiva intercultural. En esta dirección, podría ser muy útil trabajar para:

- favorecer el trabajo de redes entre los servicios y entre estos y el territorio;

- difundir y dar a conocer las buenas prácticas realizadas;

- trabajar a la superación progresiva de los servicios dedicados (servicios especiales o de emergencia para extranjeros) en vistas de la transición a la etapa de los servicios para todos, independientemente del origen del usuario;

- garantizar la presencia dentro del servicio de un equipo de colaboradores «étnicamente» mixto. Esto no siempre ocurre, y a veces casi nunca, hasta cuando el servicio utiliza constantemente a un mediador intercultural, una persona que se emplea y se encuentra en muchas estructuras.

- Invertir de manera significativa en la formación de los operadores: la competencia intercultural, la capacidad de comunicar de manera eficaz con los que pertenecen a grupos culturalmente diferentes, no forma parte de los conocimientos profesionales de la mayoría de los operadores. Se trata de actuar en la formación en diferentes niveles y, sobre todo, en aquel inicial, de manera que ellos puedan formarse, más y más, en una perspectiva internacional. Pero no debe pasarse por alto la formación continua de los profesionales que trabajan cada vez más con los inmigrantes.

Pero la perspectiva intercultural es, por su misma naturaleza, dual y requiere, no solo a la sociedad mayoritaria, sino también a los inmigrantes, que contribuyan de manera dinámica a la construcción de una sociedad inclusiva, volviendo a definir su propia identidad y afiliación. En el caso de los inmigrantes, a menudo la formación internacional se ha indicado como el mejor camino para la integración (Bonetti, Fiorucci, 2006). Esto es verdad si tenemos en consideración al menos tres aspectos centrales: la calidad de la formación proporcionada, la «globalidad de las necesidades» que los inmigrantes llevan consigo y los proyectos migratorios de los inmigrantes y de sus familias. La formación, para quienes emigran, es una síntesis de acogida y estabilización. El inmigrante acepta iniciar un camino de formación tanto para aprender a convivir en la ambigüedad de su condición, como para salir de ella. Él, o ella, empieza un nuevo proceso de identificación lingüística, socio-cultural y profesional, porque sabe que no puede aguantar vivir, por 
mucho tiempo, solo con el «bien-amparo» representado por su propia lengua, mentalidad y operatividad. El inmigrante busca la seguridad que los contenidos de la formación pueden darle - desde su primera alfabetización hasta su formación profesional(Demetrio y Favaro, 1992, p. 33).

Sin embargo, la formación por sí sola no puede solucionar los problemas de inclusión o de no inclusión de los inmigrantes: el resultado de las rutas de integración tiene que ver con las más generales políticas de integración que un país está dispuesto a poner en marcha. El caso de Italia se ha caracterizado por mucho tiempo por la falta de un modelo político y funcional diseñado de manera intencional y las medidas legislativas han seguido, esencialmente, el fenómeno migratorio, más que adelantarlo y dominarlo (Ambrosini, 2005). Se trata de un modelo no intencional que ha ido definiéndose a posteriori, tras los procesos espontáneos de inclusión informales de miles de inmigrantes en el tejido social y económico de Italia, al que corresponden, a la misma vez, tanto a grandes gestos de solidaridad por parte de los ciudadanos, asociaciones y autoridades locales, como a reacciones de rechazo y campañas de seguridad por parte de algunos miembros de la sociedad y del mundo político, así como una escasa reglamentación institucional por parte de los legisladores. A pesar de los cambios en la conducción política, una característica constante en el tiempo de la política italiana ha sido la utilización de las amnistías, como instrumento para regularizar las muchísimas situaciones en las que se encontraban miles, y a veces cientos de miles, de inmigrantes, mostrando, de manera indirecta, la inadecuación de las políticas de las cuotas de entrada anuales, como manera principal de acceso legal a Italia.

Por lo tanto, no se puede no tener en consideración el marco político más general $\mathrm{y}$, sin embargo, la formación puede volverse una prioridad para favorecer las rutas de integración de las personas inmigrantes en el tejido económico, social y cultural de los países de acogida. De hecho, cabe señalar que en algunas realidades territoriales, aunque minoritarias, se han puesto en marcha muchas iniciativas y se han experimentado proyectos interesantes en este sentido, cuyo objetivo es la integración a través de cursos de orientación, de evaluación de las capacidades, de inserción y reintegración laboral, de formación profesional y permanente y proyectos que ayudan a los extranjeros a través de procesos de alfabetización lingüística, con atención particular hacia las personas más débiles, sobre todo hacia las mujeres, que a menudo se reúnen con sus maridos en un segundo momento y que no tienen la oportunidad de integrarse en la comunidad. Sin embargo, lo que debe evitarse es el riesgo de proporcionar a los inmigrantes una formación de segunda clase. En cambio, hay que aprender a reconocer en cada extranjero a una persona que lleva consigo una historia y una memoria, que tiene una cultura y una patria, un proyecto de vida, unos conocimientos que deben valorizarse y cosas que decir; que se encuentra con diferentes problemas y tipos de dificultad que dependen de su grupo étnico, de si es hombre o mujer, chico, joven o mayor, dependiendo de su camino de migración, de si tiene o no el respaldo de una comunidad; que no necesita solo de alojamiento y comida, sino también de comunicación, de sociabilidad, de cariño y de cultura. Se trata de observar los sucesos de manera diferente respecto a cómo se vieron anteriormente, de hacerse una nueva representación y, entonces, de llevar a cabo una reestructuración cognitiva que está lejos de ser completa. Pero esta misma es indispensable si se quiere dar a los extranjeros una imagen nueva y diferente que se debe tener de ellos (Susi, 1991). 
Sin embargo, para que sea posible una transición de una condición de indiferencia o de conflictividad a otra de convivencia intercultural son necesarias personas valientes, capaces de posicionarse de manera voluntaria al límite entre la afiliación y de fomentar el mutuo conocimiento, el diálogo, la cooperación, de favorecer la sensibilización y la mediación entre los diferentes grupos; se trata de los que Alexander Langer definió como «traicioneros de la cohesión étnica», es decir personas que se comprometen en la exploración y en la superación de los límites y que son capaces de criticar también a su propio grupo de referencia, pero que nunca deben transformarse en desertores, es decir no deben sufrir una pérdida de credibilidad ante su propia comunidad de referencia. Según Langer estos «traicioneros de la cohesión étnica» son los primeros instrumentos de convivencia intercultural. En cierta medida, todos somos traicioneros de nuestras identidades originales. Y, a menudo, el gesto de la ruptura con una afiliación que se convierte en una jaula resulta ser una necesidad, a veces se delinea como una elección arriesgada, pero noble (Langer, 2007, p. 184).

\section{La perspectiva intercultural en la educación y la formación}

Como se dijo anteriormente, el momento histórico en el que vivimos está marcado por profundas transformaciones de carácter socio-económico y político y están determinadas por diferentes factores (Portera, 2006). Entre estas, el número cada vez mayor de contactos y relaciones entre personas de origen cultural diferente, que ha llevado a la planetarización de las relaciones interhumanas e interinstitucionales (Poletti, 1992). Ya son muchas las partes que afirman que la época en la que vivimos es globalizada e interdependiente. Dicho proceso se da por sentado y parece ser irreversible. Es un hecho - afirma Francesco Susi- que vivimos en una época que interconecta, pone en contacto a todas las partes del planeta. Se difunde, para decirlo de mejor manera, la conciencia de que los problemas humanos, dondequiera se originen o se realicen, también causan efectos en las regiones más remotas del planeta. Por lo tanto, se ha podido hablar, y con mucha razón, de una cultura de las interdependencias (Susi, 1995). Lo que preocupa, a pesar de todo, es la escasa concienciación del alcance «global» de los problemas del presente: nosotros vivimos en una edad planetaria - como afirmó padre Ernesto Balduccicon una conciencia neolítica (Balducci, 2005). Entonces, se trata de diseñar, predisponer y realizar itinerarios educativos y de formación que estén a la altura de los problemas del presente para entender los procesos sin tener que aguantarlos, sin que nos afecten y evitando que puedan causar el así llamado «choque de civilizaciones» (Huntington, 1996).

La educación, la escuela, la capacitación profesional y el sistema de formación en su conjunto se encuentran ante un gran reto. Si el deseo de intercambios y relaciones siempre más amplios y heterogéneos parece ser muy fuerte, es también patente la dificultad de poner en marcha un real intercambio intercultural en las sociedades multiculturales que ahora constituyen un hecho irreversible. Por consiguiente, es necesario elaborar soluciones adecuadas para una gestión consciente y equilibrada de las diferencias y de las diversidades. Incluso hoy en día, por el contrario, el tema de la inmigración, a pesar de estar en el centro de continuas discusiones y debates que condicionan muchísimo las campañas electorales y las elecciones políticas, ha despertado solo parcialmente un debate serio y profundo por parte de las instituciones y de las fuerzas políticas sobre los temas de la educación, de la formación y de la escuela. 
A pesar del hecho de que los fenómenos migratorios marcan Italia desde hace treinta años, se puede afirmar que hoy en día todavía no se tienen en particular consideración las necesidades formativas y culturales de los inmigrantes (Susi, 1991), puesto que la atención se centra casi exclusivamente en aquellas esenciales (casa, salud, trabajo). Las necesidades formativas y culturales no son «algo más», un lujo que debe darse a los inmigrantes cuyas necesidades primarias estén satisfechas. Estas, se encuentran - como demuestran las investigaciones - en cada etapa de la experiencia de inmigración y condicionan sus desarrollos y resultados, dependiendo de las respuestas que reciben (Susi, 1995). Para enfrentarse a los problemas que vienen de la presencia de personas que vienen de países diferentes, es también necesaria una fuerte respuesta educativa.

La educación y la formación son las primeras y más importantes herramientas, puesto que permiten la socialización de los miembros de la comunidad y la transmisión de la herencia cultural acumulada por el hombre. Las experiencias, los estudios, las investigaciones y las reflexiones sobre el tema de la educación intercultural en Italia y en Europa han llevado a la concienciación de la necesidad de crear un encuentro productivo entre diferentes conocimientos que trabajen a la definición de una «pedagogía intercultural». Los diferentes enfoques que se han intentado demuestran que hablar conscientemente de «educación intercultural» implica la necesidad de ampliar el debate a través de la ayuda de muchas más disciplinas, como la lingüística, la psicología, la pedagogía comparada, la demografía, la antropología cultural, etc. y que es necesario dirigirse hacia todos los estudiantes, autóctonos e inmigrantes.

No se trata tanto, como se oye a menudo de manera enfática y retórica, de prestar atención a las culturas en sí mismas, sino de prestar atención a las personas que las llevan consigo. Por lógica, no son las culturas las que se encuentran, sino las personas, que vuelven a elaborar de manera única y original su relación con los sistemas culturales de origen. Por eso resulta peligrosa una interpretación de la cultura como si fuera algo estático e inmodificable. La cultura es un conjunto de referencias, símbolos, creencias, que recibimos como herencia, pero también un camino individual nuevo que tiene el carácter de la imprevisibilidad y de la elección. La cultura - todas las culturas - es viva y dinámica, se pone en un proceso de continuo cambio que se origina a través de intercambios, encuentros, la aceptación de distintas contribuciones y también la defensa de sus propios principios y referencias. La cultura de cada uno de nosotros se construye y se modifica siguiendo adelante y se convierte en nuestra historia, gracias a las elecciones y a las variaciones individuales, que nos vuelven, en algunos aspectos, parecidos a aquellos que se encuentran en nuestro grupo y en nuestra familia, pero también extremadamente diferentes respecto a cada individuo (Favaro y Fumagalli, 2004). Toda persona, empezando por un determinado sistema de valores, de conocimientos y de comportamientos dentro de los que nace y crece, elabora una relación individual y original con su propia «cultura de pertenencia». Además, dicha relación está condicionada por el entorno de pertenencia, por la clase social, por el género, por la educación, y de manera más general, por las condiciones sociales, culturales y económicas.

La tarea que nos espera, en los próximos años, es exactamente esta: no solo reflexionar en las culturas, sino, una vez más, directamente en el hombre (Bettini, 1992, p. 15). La pedagogía intercultural es, en primer lugar, una «pedagogía relacional» cuyo objetivo, por definición, es el de enfrentarse al problema de las relaciones entre miembros de 
diferentes culturas, a vistas de la mutua aceptación y del respeto (Desinan, 1996, p. 17). Se trata de una orientación del pensamiento que se traduce en ideas, acciones, decisiones, pero también es una estrategia educativa, la detección de una práctica formativa que debe, además, analizar de manera profundizada el concepto de «integración cultural», concepto en el que podrían esconderse las ideas de «asimilación» y de «segregación».

La educación intercultural se configura como la respuesta en cuanto a prácticas de formación a los retos planteados por el mundo de las interdependencias; esta es un proyecto educativo intencional que cruza de manera transversal todas las disciplinas que se enseñan y cuyo objetivo es el de modificar las percepciones y los hábitos cognitivos con los que generalmente se representan tanto a los extranjeros como el nuevo mundo de las interdependencias (Susi, 1995). La pedagogía intercultural debe establecer «cuáles» valores deben transmitirse y «cómo» transmitirlos y es en este sentido que se transforma en didáctica, a través de actividades tanto cognitivas como de contacto de experiencias con diferentes enfoques. De hecho, se habla de «didácticas interculturales»: aquellas que se centran en los puntos de vista o en los lugares de vida (cuando se quiere mostrar la pluralidad de las concepciones cognitivas que se refieren a componentes culturales que permiten la comparación entre el nosotros y el vosotros), en la expresividad (sobre las diferencias en el arte, en el mobiliario, en la música, en el folclore, etc.) en el malestar del otro (sobre la inmigración y sobre sus implicaciones locales e internacionales, pero también sobre la pérdida de sus propias raíces y sobre la investigación de una nueva identidad), en la semejanza (sobre todo lo que nos vuelve iguales en términos de los derechos y deberes, o sobre los que podrían definirse los arquetipos de la metacultura) (Demetrio y Favaro, 1996). Estas didácticas, cuyo objeto está constituido por las relaciones mentales, físicas, interpersonales y de experiencias que se crean precisamente entre los individuos de diferente pertenencia, más que en la información, hacen hincapié en los procesos que nos permiten apoderarnos de los conocimientos con vistas a «formar a la reciprocidad». Pero lo que parece ser aún más urgente es profundizar una reflexión seria y estricta sobre las didácticas interculturales de las disciplinas y de los conocimientos y sobre las experiencias que se han llevado a cabo, en este sentido, hasta hoy en día. Por lo tanto, se vuelve necesario volver a considerar los planes de estudio formativos y los estilos de comunicación que marcan el encuentro como ocasión de mutuo descubrimiento, así como los programas, los libros de texto y los materiales didácticos para corregir su orientación monocultural (Brunelli, Cipollari, Pratissoli, 2007; Catarci, 2004; Fiorucci, 2008; Luatti, 2009).

Entonces, la educación intercultural no tiene una tarea fácil ni de corto plazo, puesto que implica una revisión de los actuales conocimientos que se enseñan en las escuelas y porque la educación intercultural no es una nueva disciplina, que se añade a las demás, sino un punto de vista, una perspectiva diferente con la que ver los conocimientos que se enseñan en la actualidad (Fiorucci 2008). El origen de la educación intercultural debe relacionarse al desarrollo de los fenómenos migratorios y, sin embargo, hoy en día ha abandonado el terreno de la educación especial que se refiere a un grupo social específico, volviéndose un enfoque pedagógico innovador para la renovación de los planes de estudio y de la pedagogía en general. 


\section{Referencias bibliográficas}

AMBROSINI, M. (2005): Sociologia delle migrazioni. (Bologna: Il Mulino).

BALDUCCI, E. (2005): L'uomo planetario, (Firenze: Giunti).

BETTINI M. (ed.) (1992): Lo straniero ovvero l’identità culturale a confronto. (RomaBari: Laterza).

BONETTI S. y FIORUCCI M. (eds.) (2006): Uomini senza qualità. La formazione dei lavoratori immigrati dalla negazione al riconoscimento. (Milano: Guerini).

BRUNELLI C., CIPOLLARI G. Y PRATISSOLI, M. (2007): Oltre l'etnocentrismo. I saperi della scuola al di là dell'Occidente. (Bologna: EMI).

CAMPANI, G. (2008): Dalle minoranze agli immigrati. La questione del pluralismo culturale e religioso in Italia. (Milano: Unicopli).

CATARCI, M. (2004): All'incrocio dei saperi. Una didattica per una società multiculturale. (Roma: Anicia).

COMISIÓN EXTRAORDINARIA PARA LA PROTECCIÓN Y EL FOMENTO DE LOS DERECHOS HUMANOS DEL SENADO DE LA REPÚBLICA ITALIANA (Legislatura XVI) (2011): Rapporto conclusivo dell'indagine sulla condizione di Rom, Sinti e Caminanti in Italia. (Roma).

DEL BOCA, A. (2005): Italiani, brava gente? Un mito duro a moriré. (Vicenza: Neri Pozza).

DEMETRIO, D. y FAVARO, G. (1992): Immigrazione e pedagogia interculturale. (Firenze: La Nuova Italia).

DEMETRIO, D. y FAVARO, G. (1996): Bambini stranieri a scuola. Accoglienza e didattica interculturale nella scuola dellinfanzia e nella scuola elementare. (Firenze: La Nuova Italia).

DESINAN, C. (1996): Orientamenti di educazione interculturale. (Milano: Franco Angeli).

FAVARO, G. y FUMAGALLI, M. (2004): Capirsi diversi. Idee e pratiche di mediazione interculturale. (Roma: Carocci).

FIOURUCCI, M.. (ed.) (2008): Una scuola per tutti. Idee e proposte per una didattica interculturale delle discipline. (Milano: Franco Angeli).

FLEXNER, A. (2013): La utilidad de los conocimientos inútiles, en Ordine, N. La utilidad de lo inútil. Manifiesto. Con un ensayo de Abraham Flexner. (Barcelona: Acantilado).

FUNDACIÓN MIGRANTES (2015): Rapporto Italiani nel Mondo 2015. (Todi (PG): TAU Editrice). 
HUNTINGTON, S. P. (1996): El choque de civilizaciones y la reconfiguración del orden mundial. (Buenos Aires - Barcelona - México: Paidós Iberica).

IDOS - UNAR (2015): Dossier statistico sull'immigrazione 2015. (Roma: Idos).

LEY n. 482 del 15 de diciembre de 1999 llamada «Normas en materia de tutela de las minorías lingüísticas históricas», publicada en la Gazzetta Ufficiale del 20 de diciembre de 1999 n. 297.

LERNER, G. (2007): Tu sei un bastardo. (Milano: Feltrinelli).

LUATTI, L. (ed.) (2009): Educare alla cittadinanza attiva. Luoghi, metodi, discipline. (Roma: Carocci).

MINISTERIO DELINTERIOR ITALIANO, OFICINACENTRALPARA LOS PROBLEMAS EN LAS ZONAS FRONTERIZAS Y DE LAS MINORÍAS ÉTNICAS. (1994): Primo rapporto sullo stato delle minoranze in Italia. Roma.

MIUR-ISMU (2015): Alunni con cittadinanza non italiana. Tra difficoltà e successi. Rapporto nazionale A.s. 2013/2014. Quaderni Ismu 1/2015. (Milano: Fondazione ISMU).

MORIN, E. (2001): Los siete saberes necesarios para la educación del futuro. (Santillana: Unesco).

NUSSBAUM, M. C. (2005): El cultivo de la humanidad: una defensa clásica de la reforma en la educación liberal. (Barcelona: Paidós Ibérica).

NUSSBAUM, M. C. (2010): Sin fines de lucro. Por qué la democracia necesita de las humanidades. (Buenos Aires - Madrid: Katz editores).

ORDINE, N. (2013): La utilidad de lo inútil. Manifiesto. Con un ensayo de Abraham Flexner. (Barcelona: Acantilado).

POLETTI, F. (ed.) (1992): L'educazione interculturale. (Firenze: La Nuova Italia).

PORTERA, A. (2006): Globalizzazione e pedagogia interculturale. (Trento: Erickson).

SANTERINI, M. y REGGIO P. (eds.). (2007): Formazione interculturale: teoria e pratica. (Milano: Unicopli).

SUSI, F. (1991): I bisogni formativi e culturali degli immigrati stranieri. La ricercaazione come metodologia educativa. (Milano: Franco Angeli).

SUSI, F. (1995): L'interculturalità possibile. L'inserimento scolastico degli stranieri. (Roma: Anicia).

TODOROV, T. (2008): El miedo a los bárbaros, más allá del choque de civilizaciones. (Barcelona: Galaxia Gutenberg/Círculo de Lectores). 